\title{
Carrier technology/humic additives to upgrade biological water treatment
}

\author{
V. Jirku, J. Masak \& A. Cejkova \\ Institute of Chemical Technology in Prague, Prague, Czech Republic
}

\begin{abstract}
The limitations of wastewater bio-treatments have challenged cell biologists and bioengineers to search for new approaches to upgrade the function and stability of these processes. In this connection, we have been trying to find a widely applicable approach to optimize the clean up efficiency through enhanced capability of fungal biodegraders both to tolerate pollutant toxicity or stressing conditions of the environment and to utilize less accessible pollutants. This paper reports some findings enabling aimed applications of fungal biofilms and humic additives.
\end{abstract}

Keywords: cell attachment, biofilm, humic acid, biodegradation, wastewater treatment.

\section{Introduction}

Exclusive application of attached biodegraders and a detailed knowledge of cell attachment effect appear to offer a large scale of opportunities not only to upgrade bioreactor (permeable bio-barrier) design, but also to reduce markedly the amount of treatment equipment as well as to enhance the spectrum of biodegradation activity through flexible co-attachment of complementary modulated cell populations, among others. Unlike microbial cells freely dispersed in an aqueous phase, attached (biofilm) cells associated with surfaces develop spatial relationship to each other that permit interactions approaching those of multicellular organisms [1]. In mixed species biofilm communities this provides intra-population variations as well as inter-population interactions offering technologically significant phenotype modulations. This cell attachment effect, together with now recognized capacity of microbial cells to sense and respond to physical contacts, is a potential tool to optimize the biodegradative 
function and behavior of water treatments microflora through changes in metabolic activity, survival and fitness of microbial population. In this context, the knowledge obtained is shown not only in view of the application of fungal biodegraders, but also in view of one of the bottlenecks of water bio-treatment systems, i.e. an insufficient capability of their microflora to take up hydrophobic pollutants.

\section{Material and methods}

The organisms used: Fusarium proliferatum (an acetone degrader), Candida maltosa (a phenol degrader) and Rhodotorula mucilaginosa (a naphthalene and phenanthrene degrader) were obtained through the E! 2113 (WABIS) strain selection programme. The covalent attachment of yeast cells via glutaraldehyde amino spacers to a physiologically inert hydroxyalkyl methacrylate gels has been shown as a suitable method to simulate the conditions of natural (multipoint) cellular bioadhesions. Keramzit particles (AGRO Ltd, Czech Republic) were colonized, respectively, by yeast and mycelial cells forming natural biofilms. Ultrasonically cleaned (Sonorex, Bandelin, Germany) Na-silica glass (EcoGlas Ginzel, Czech Republic) was used as a standard carrier material for the cell adhesion (attachment) studies; glass modification was performed, respectively, through $3 \mathrm{~h}$ exposure to $6 \mathrm{M} \mathrm{HCl}$ at $100^{\circ} \mathrm{C}$ and by silanization through $\gamma$-aminopropyltriethoxysilan hydrolysis. Glass hydrophobicity was assayed as contact angle of water drop on glass surface [2]. The $90 \times 8 \times 4 \mathrm{~mm}(1 \times \mathrm{w} \times \mathrm{h})$ flow cell of effective volume $2.88 \mathrm{~mm}$, used for flow rate effect experiments, was made of stainless steel and polymethylmethacrylate. The biofilm carrier used throughout here is a sandwich-like foil with $40 \mu \mathrm{m}$ polypropylene core covered with $12 \mu \mathrm{m}$ polyester outer layer (Steriking ${ }^{\circledR}$, WIPAK MEDICAL, Finland). Microbial attachment to the carrier was performed under standard conditions of the carrier-cell population (statical / flow) contact. Resistance experiments involved growing suspended and covalently attached cells exposed, respectively, to individual toxic compounds. The used concentration of the agent was that found as inhibiting (MIC) the suspended cell growth and reproduction. The hyperosmotic shock was performed through the immediate mixing of the culture medium with a quantity of pure glycerol determined according to Norrish's equation [3] to obtain final $a_{w}=0.900,0.800$, and 0.700 . The control was carried out using the same ratio to dilute the culture medium with a waterglycerol solution $\left(a_{w}=0,990\right)$. The suspended or attached cell populations were allowed to equilibrate for $20 \mathrm{~min}$ under the culture conditions $\left(28^{\circ} \mathrm{C}, 90 \mathrm{rpm}\right)$ before any measurement was initiated under osmotic stress. Free and bound cell dry weights were determined on the basis of nitrogen content assayed by Kjeldahl's method. Degradative function of yeast and mycelial biofilms was investigated using jacketed, tubular reactor $(30 \mathrm{~cm}$ inner diameter; $70 \mathrm{~cm}$ length), enabling temperature $\left(18^{\circ} \mathrm{C}\right)$ control and a countercurrent, continual circulation of media $(\mathrm{pH} 4.7)$ and air. Degradative function of suspended cells was tested using a bioreactor (Braun Biotech International, Germany) with an operating volume of $2.0 \mathrm{l}$, and temperature $\left(18^{\circ} \mathrm{C}\right), \mathrm{pH}(4.7)$ and rotation speed 
(120 rpm) control. Marker pollutant was used as a sole source of carbon in the minimal salts medium. LUCIA 3.52 for Windows 95 was used for the mycelial biofilm image processing. Yeast artificial and natural biofilm was coated with gold layer (Balzers Union SCD-040, Germany) and observed on a JXA-840A Electron Probe Microanalyzer (JEOL, Japan).

\section{Results and discussion}

To tentatively clarify the nature and scope of (fungal) cell attachment effect, a wide range of markers has been used to compare suspended and attached (viable) yeast cells. Using different monolayers of attached yeasts (as for instance Fig. 1), the phenotypes of suspended and attached cells were compared. The data obtained (not shown) proved no significant differences regarding tested markers of primary metabolic activity. Attached yeast cells show, however, increased concentrations of mannan and B-1,3-, B-1,6-alkali-insoluble glucans as well as a higher intensity of biosynthesis of all main lipids, a higher proteosynthetic capacity, and a decreased level of glycogen and trehalose. A comparison of total content of cell wall proteins, lipids, and amino sugars in suspended and attached cells shows that the cell walls of attached unicellular fungi contain increased amounts of each component. A different composition of mannoproteins in the walls of attached cells was detected in parallel. A comparison of the sterol / phospholipid ratio, between suspended and attached cells, exhibited no notable difference, however, cell attachment stimulates an increase in the total amounts of both of these lipids. Moreover, cell attachment stimulates a significant shift up in the excretion patterns of acid phosphatase and B-glucanases; an enhancement of the extracellular production of alkaline protease, specifically induced by four different starvation conditions, was found as well.
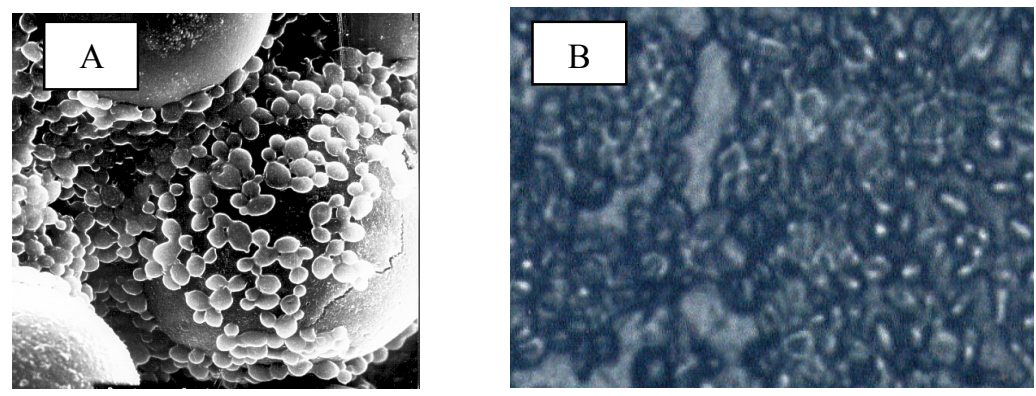

Figure 1: Scanning electron micrograph of covalently attached (A), on Steriking ${ }^{\circledR}$ membrane attached (B) monolayers of growing yeast cells.

The aforementioned results encouraged the idea that the cell attachment, stimulating compositional changes in the cell surface structures, could allow us to manipulate, among others, the susceptibility of fungal biodegraders both to the 
pollutants and extracellular conditions damaging these structures. In this connection, the uniform pattern of attached cells resistance to respective effect of acetone, benzene, dimethyl sulfoxide, ethanol, ethylene glycol, phenol, mercaptoethanol, dithiothreitol and 5-bromo-6-azauracil proves that the yeast cell attachment induces a pleiotropic tolerance to cytotoxic agents (Fig. 2). Similarly, the effect of reductions in water activity $\left(\mathrm{a}_{\mathrm{w}}\right)$ on the growth activity of the suspended and attached yeasts was found different and indicating that attached yeast cells can tolerate a growth inhibiting (osmotic) effects of the environment (Fig. 3). Moreover, attached (more resistant) cell populations show an uniform increase in the proportion of saturated and a decrease in the proportion of unsaturated fatty acids, as well as an increase in the content of three phospholipids and ergosterol The attached (more osmo-resistant) yeast populations show an uniform decrease in the trehalose level (not shown). The results obtained support the rising hypothesis that a multipoint contact sensing of fungal cell surface brings significant modulation of cellular structures, functions and properties. The long-term stability of compositional alterations and the resistance observed suggest that their development is not stimulated by transient stimuli in the cell surface microenvironment. The paucity of information about the existence of a transductive system participating in a cell response to cell (physical) contacts, and a range of diverse effects potentially acting in an attached cell microenvironment, make any clear-cut explanation of the above phenomenon impossible.
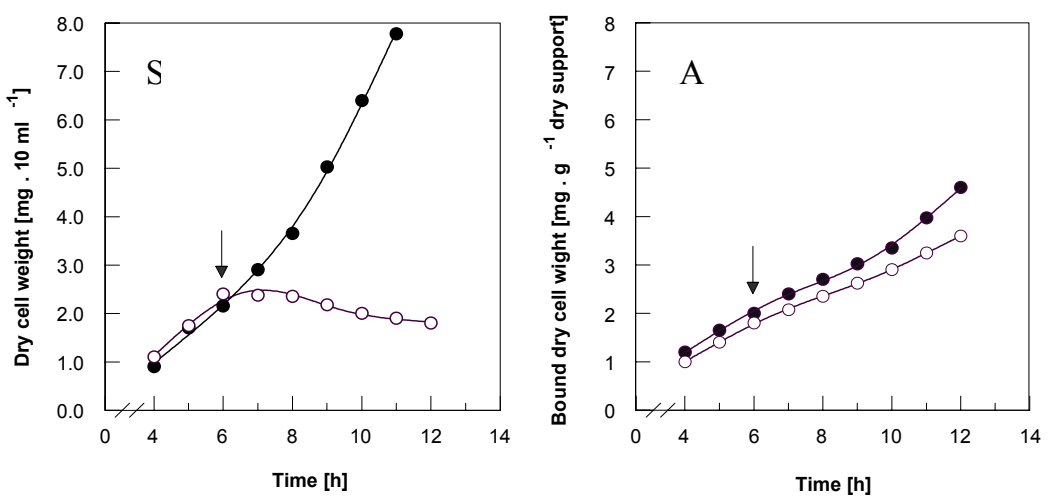

Figure 2: Uniform pattern of growth inhibition of suspended (S) and covalently attached (A) yeast cells exposed to the effect of different cytotoxic agents (see Results and discussion). ( $\downarrow$ ) added, respectively; $(\bullet)$ control cell population was cultivated without inhibitor. 

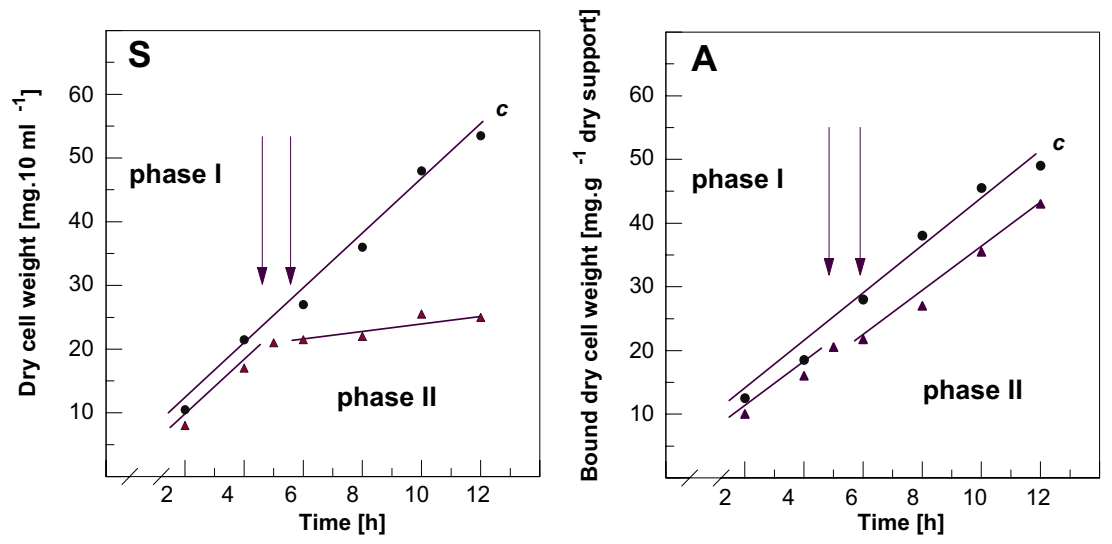

Figure 3: Growth inhibition of suspended (S) and covalently attached (A) yeast cells exposed to low hydrated media $\left(\mathrm{a}_{\mathrm{w}}=700\right)$. ( $\left.\downarrow \downarrow\right)$ glycerol added; (c) control (see Material and methods).

Nevertheless, the detected compositional changes must primarily affect (enhance) the rigid membrane / wall framework as well as the biochemical functions that are connected with these structures. The technological potential of the protective effect of fungal cell attachment illustrates a column reactor simulation of phenol and acetone removal performed, respectively, by $C$. maltosa and $F$. proliferatum natural biofilm (Fig. 4).
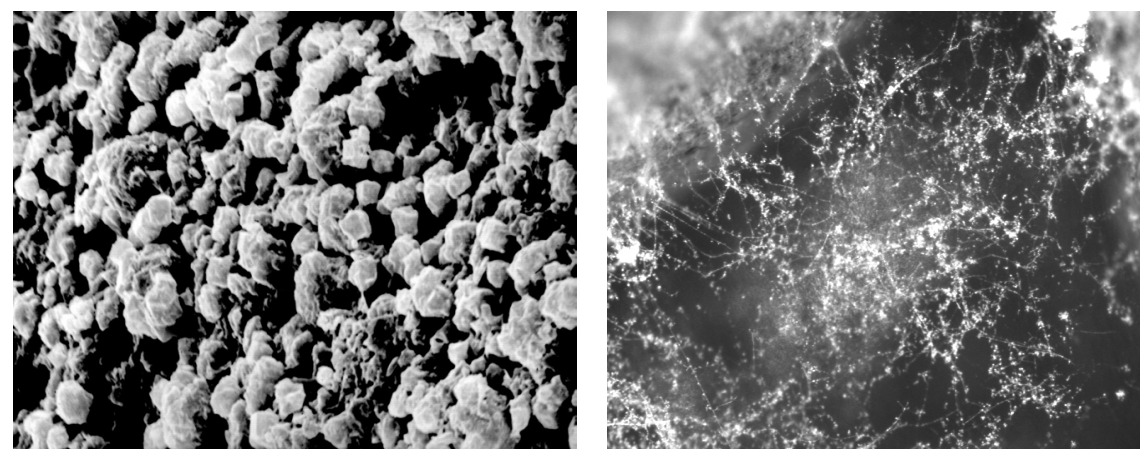

Figure 4: Candida maltosa (A) and Fusarium proliferatum (B) biofilm formed on a keramzit particle after $72 \mathrm{~h}$ of the reactor cultivation.

In comparison with suspended biodegraders this model shows that the removal efficiency is significantly enhanced if natural biofilms are used (Figs. 5 and 6). 


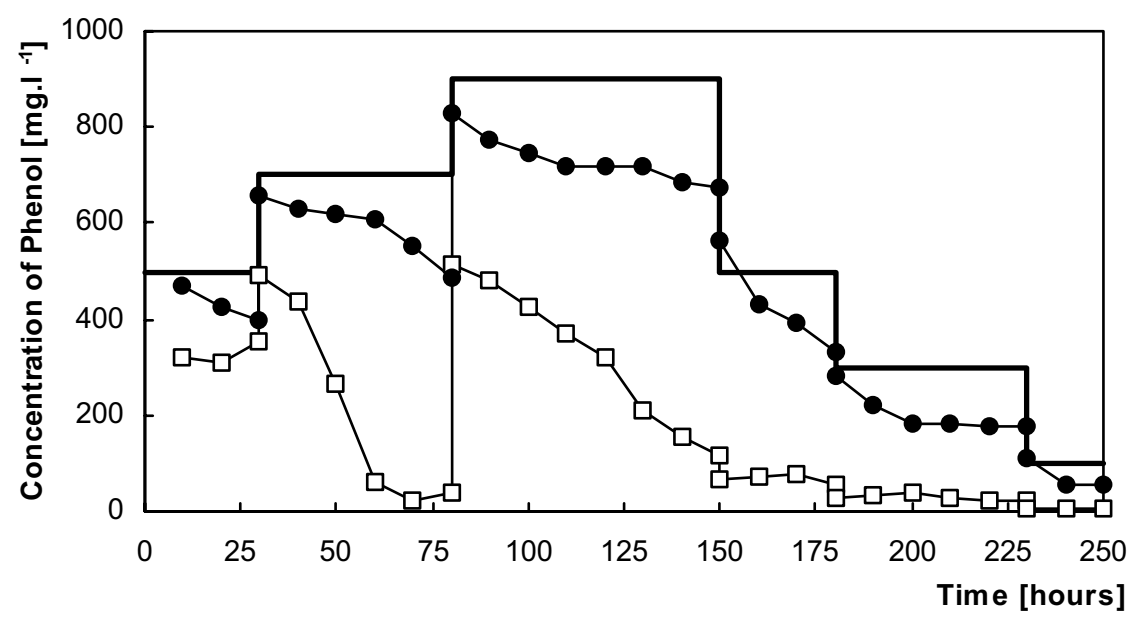

Figure 5: Changes in outlet phenol concentration in attached ( $\square$, keramzit) and suspended $(\bullet)$ population of $C$. maltosa as a function of time and input phenol concentration (一); (flow rate $1 \mathrm{ml} \mathrm{min}^{-1}$ ).

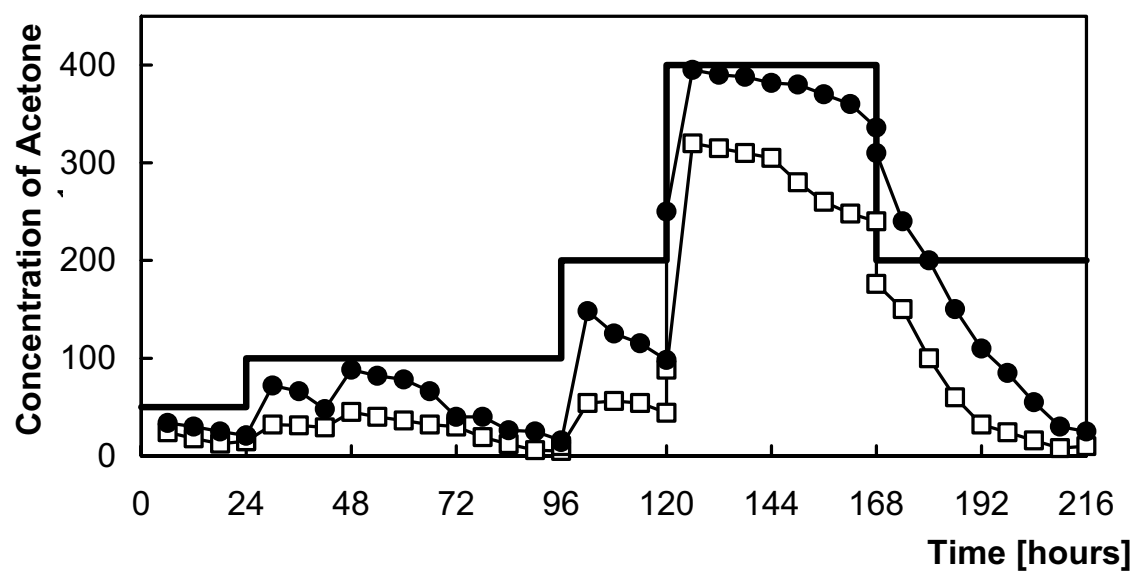

Figure 6: Changes in outlet acetone concentration in attached ( $\square$, keramzit) and suspended $(\bullet)$ population of C. maltosa as a function of time and input acetone concentration (一); (flow rate $1 \mathrm{ml} \mathrm{min}^{-1}$ ).

Moreover, the inhibitory effect of the pollutant concentration, which almost or significantly inactivate the reactor degradative function, is reversible, if the level of pollutant in the recirculating medium is considerably lowered within next $12 \mathrm{~h}$., and the physiological adaptation of both biofilms to each higher concentration of model pollutant (or a higher flow rate of the media) is 
accompanied with a transient suppression of its uptake. In addition, quantitative data obtained for yeast biofilm formation under different flow rates of growth medium suggest that the intensity of fungal biofilm formation can be controlled by medium flow rate as well (not shown). Limitations in the mass-transfer rate of a phase, in which biodegradation can occur is widely accepted as the cause of some pollutants persistence. Therefore to enhance their bioavailability and accordingly biodegradation, the search for additives affecting the pollutant / biodegrader contact is wanted. The similarities between the effect of micelle forming surfactants and humic acid on the surface tension has prompted the suggestion that humic acid is a potentially useful agent enhancing the accessibility of some pollutant to be degraded. In this connection, the use of humic acids was investigated with a prerequisite that fungal degraders can tolerate humic compounds and take up above pollutants to degrade them more efficiently and less costly to meet clean up standards. In view of the fact that natural biofilm formation is always determined by complex regulation of surface attachment and consequent biofilm maturation, another field of our research has been focused on the effect of the cell surface hydrophobicity on the (fungal) cell adhesion correlating this effect with the influence of environmental stressors and carrier hydrophobicity. In practical terms, the biofilm formation detected was found to be affected in a complex manner by carrier modification, cell type as well as by physiologically acting factors of extracellular environment. In Figs. 7 and 8 is shown that, e.g. for materials and cells that are mutually repellent, the effect of some stressors can enhance the adhesion capacity of microbial cell.

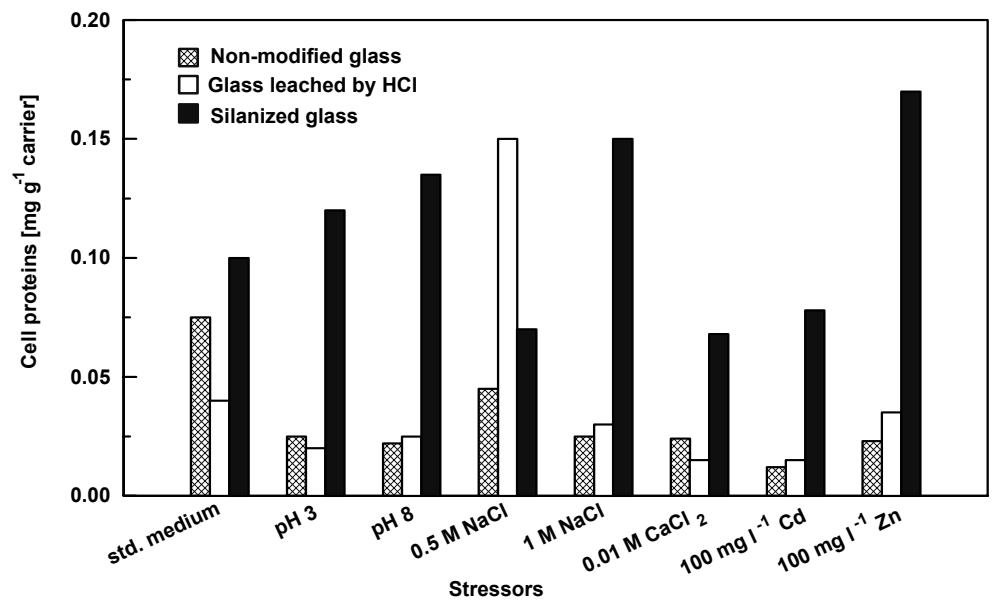

Figure 7: Effect of stressors on the adhesion of Candida maltosa to modified glass.

As is evident from Fig. 9, the presence of humic acid evokes a considerable enhancement of yeast capacity to utilize, respectively, naphthalene and phenanthrene as a sole source of carbon; (yeast biodegraders were found to be not capable of utilizing humic acids as a sole source of carbon). The parallel 
results, indicating that some effects of humic substances on yeast cell could consist in their ability to aggregate on the cell surface (not shown), as well as different chemical nature of individual humic compounds, suggest that any upgrading of biodegradation efficiency through a humic compound effect will be determined, among others, by the binding capacity of this compound in the aqueous phase.

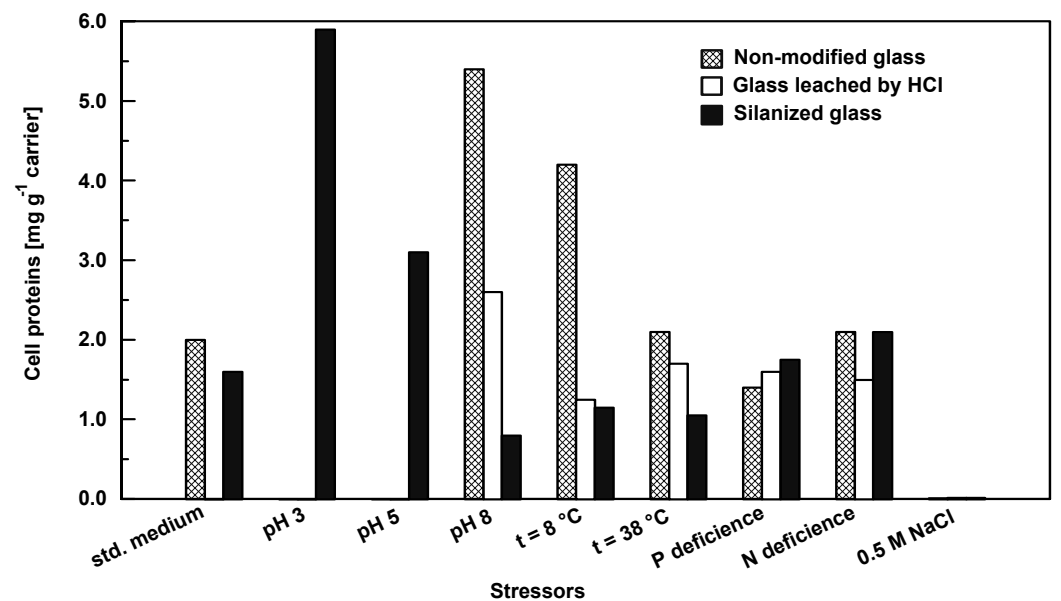

Figure 8: Effect of stressors on the adhesion of Fusarium proliferatum to modified glass.

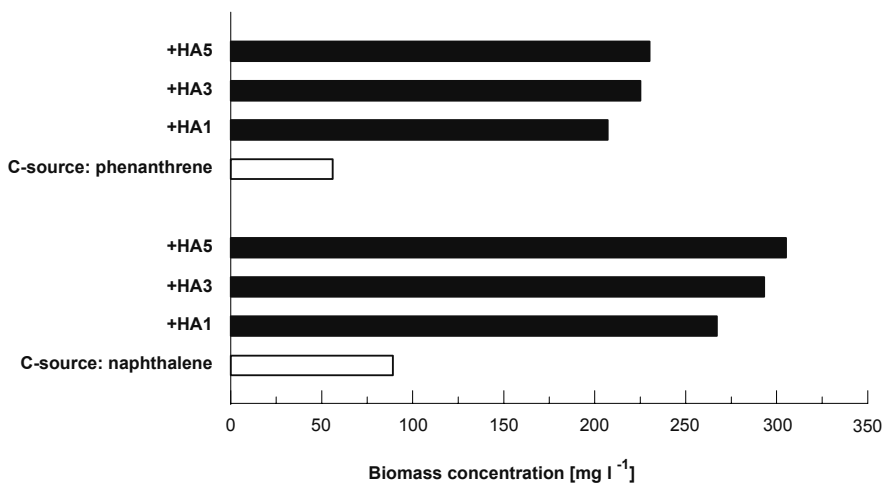

Figure 9: $\quad$ R. mucilaginosa biomass yield in the presence ( $\square$ ) and absence ( $\square$ ) of respective humic acids (HA1, HA3, HA5) and naphthalene / phenanthrene as sole source of carbon; (batch/semi-continuous) reactor growth; HA 0.05 g..$^{-1}$. 
In view of the fact that humic acid solutions are polydisperse, and the interaction of molecular segments of different sizes leads to a degree of intermolecular aggregation that further augments some of their properties, including binding (complex forming) capacity [4], the analysis of sorption potential of different humic acids was performed [5] and the equilibrium binding constant $\mathrm{K}_{\mathrm{b}}$ was quantified for naphthalene and phenanthrene, respectively, using the below equations:

$$
K_{b}=\frac{[P A H-H A]}{[P A H][H A]}
$$

where $[\mathrm{PAH}],[\mathrm{HA}],[\mathrm{PAH}-\mathrm{HA}]$ are the concentrations of komplex entering components

Assuming that the intensity of fluorescence is directly proportional to the concentration of polyaromatic hydrocarbon, then the equation might be written as

$$
\frac{F_{0}}{F}=1+K_{b}[H A]
$$

where $\mathrm{F}_{0}$ or $\mathrm{F}$ is the fluorescence intensity measured in the presence / absence of a humic acid.

The differences in the values of calculated $K_{b}$ constants (Table 1) correspond to different effect of the humic acids tested (Fig. 9).

Table 1: $\quad$ Humic acid equilibrium binding constants $\left(\mathrm{K}_{\mathrm{b}}\right)$.

\begin{tabular}{ccc}
\hline \multirow{2}{*}{ Humic acid } & \multicolumn{2}{c}{$\mathrm{K}_{\mathrm{b}}$} \\
\cline { 2 - 3 } & naphthalene & phenanthrene \\
\hline HA 1 & $4.4 \pm 1.0 \times 10^{4}$ & $9.4 \pm 0.1 \times 10^{4}$ \\
HA 3 & $3.0 \pm 0.5 \times 10^{4}$ & $6.2 \pm 0.1 \times 10^{4}$ \\
HA 5 & $2.9 \pm 0.1 \times 10^{4}$ & $3.1 \pm 0.5 \times 10^{4}$ \\
\hline
\end{tabular}

\section{Conclusions}

Wastewater treatment systems R\&D, implementing above approaches on a module basis (biodegraders selection / carrier choice - biofilm formation / biofilm function-additives / reactor loads) has been successfully applied in the Czech Republic (Aquatest SG - contact e-mail: sima@aquatest.cz), using a cascade of reactor vessels loaded, respectively, with organic and inorganic carriers colonized by specifically acting (fungal) degraders. 


\section{Acknowledgement}

This study was supported by grant no. E! 3100 (CAWAB) from the EUREKA program.

\section{References}

[1] Allison, D.G., Gilbert, P., Lappin-Scott H.M. \& Wilson, M. Community structure and co-operation in biofilm. Cambridge University Press: United Kingdom, 2000.

[2] Mozes, N. \& Rouxhet, P. G. Methods for measuring hydrophobicity of microorganisms. J. Microbiol. Methods 6, pp. 99-112, 1987.

[3] Norrish R. S. An equation for the activity and equilibrium relative humidities of water in confectionary syrups. J. Food Technol. 1, pp. 25$39,1996$.

[4] Wandruszka, R., Ragle, C. \& Engebretson, R. (1997) The role of selected cations in the formation of pseudomicelles in aqueous humic acid. Talanta 44, pp. 805- 809, 1997.

[5] Gauthier T. D., Shane E. C. and Guerin W. F. (1986) Fluorescence quenching method for determining equilibrium constants for polycyclic aromatic hydrocarbons binding to dissolved humic materials. Environ. Sci. Technol. 20, pp. 1162-1166, 1986. 